\title{
'Sex and crime' in evolution - why sexuality was so successful ${ }^{\dagger}$
}

\author{
Ingo Schubert* \\ Leibniz Institute of Plant Genetics and Crop Plant Research (IPK), \\ Corrensstrasse3, D-06466 Gatersleben, Germany
}

(Received 9 December 2010, accepted 12 January 2011)

\begin{abstract}
Components of sexuality have fore-runners already in prokaryotes, for instance conjugation, recombination- repair and the molecular constituents needed for nuclear division. For eukaryotes, the basic and predominant mode of propagation is via sexuality, although it is highly complex and costly. Many interactions between individual cells are detrimental for one partner and might be considered as a 'criminal' act performed by the active partner. For instance, the irreversible and non-reciprocal processes of phagocytosis or endocellular parasitism but also the irreversible, asymmetric and sustainable endosymbiosis with a benefit bias in favour of the active partner represent such events. Contrary to this, sexuality in general represents an indirectly reversible, reciprocal, sustainable (by reiteration) and mutually beneficial interaction between equal-ranking cells. After fertilization, a doubled set of genetic information protects against loss of essential genes, while the haplophase allows ridding lethal mutations. Resorting of parental chromosome sets, recombination between homologous chromosomes during meiosis, and new combination of alleles during fertilization, mediate a high genetic variability at a minimum risk of deleterious variants, thus promoting evolutionary adaptability.
\end{abstract}

Key words: eukaryotes, evolution of sexuality, cellular interactions, generation of genetic variability, recombination

The fascinating diversity, high complexity and evolutionary success of eukaryotes is based on a few prerequisites. One obviously was the ability to expand genome size on a large scale. For example, duplication of partial or whole genomes allows a continuous evolution of genes from duplicates of pre-existing ones (Ohno, 1970). Toleration of significant amounts of dispersed repetitive sequences that accompany genome expansion (such as for instance retroelements) requires linear chromosomes. Circular prokaryotic genomes apparently cannot easily expand by gaining (dispersed) redundant sequences. Due to the 3'-5' complementary strands of the double helix, in circular prokaryotic genomes, recombination between directed repeats may split the ring-shaped genome by appropriate resolution of the Holiday junction(s) into two rings and lead subsequently to a lethal loss of one of the products, if segregation into daughter cells is not concerted, because of the haploid character of most prokaryotes. Linear chromosomes, in turn, have to be

Edited by Hiroshi Iwasaki

* Corresponding author. E-mail: schubert@ipk-gatersleben.de

$\dagger$ This paper is dedicated to the memory of my teacher and friend Professor Rigomar Rieger who passed away on September $14,2010$. protected by specific telomere structures against replication-mediated shortening. All known DNA-polymerases need 3'-end RNA primers to start replication, which at the 5'-chromosome ends leave a gap after removal; this endshortening is compensated in most eukaryotic organisms by telomerases which add telomere repeat sequences to the chromosome ends. Linear chromosomes also are sensitive to exonuclease activity, and illegitimate recombination, when linear chromosome ends are mistakenly treated as double strand breaks. Telomeres, centromeres and mitotic nuclear division together mediate faithful segregation of replicated identical chromosomes into daughter nuclei and were necessary prerequisites in the evolution of stable eukaryotic genomes. It is still not clear whether the eukaryotic nucleus evolved by subcellular compartmentation (Margulis and Sagan, 1986) or as a product of endosymbiosis (Mereschkowsky, 1905), e.g. by absorption via recombination of the circular genome of a host cell into the linear genome of an endosymbiont (Schubert, 1988). What else made eukaryotes the favourite of evolution? 
Sex as a compelling mode of cellular interaction Upon their origin, eukaryotic cells, before and after the emergence of multicellularity, had several options to interact with other cells, prokaryotic or eukaryotic ones.

Most interactions are asymmetric and irreversible (Fig. 1). Either one cell engulfs and phagocytises the other, or one cell is penetrated by another, parasitic one and is eventually destroyed and digested.

A further type of irreversible and asymmetric interaction is sustainable but more rare (Fig. 1). It is endosymbiosis that led to mitochondria, apparently a primary event very early in eukaryote evolution, to plastids in algae and higher plants, and possibly to other eukaryotic organelles including microtubule-organizing centres (Margulis and

\section{Phagocytosis}
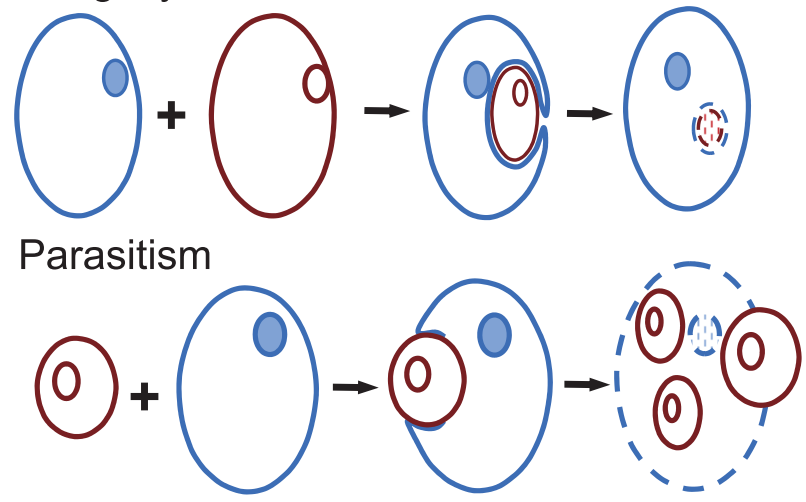

Endosymbiontic organelle formation

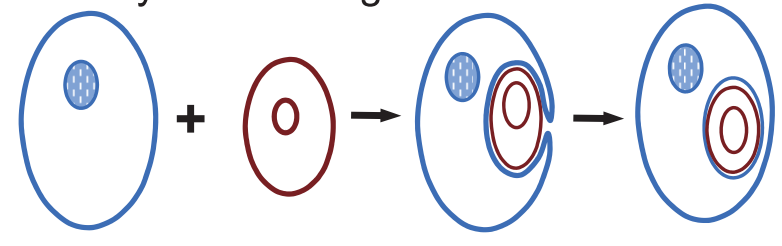

\section{Endoparasitism}

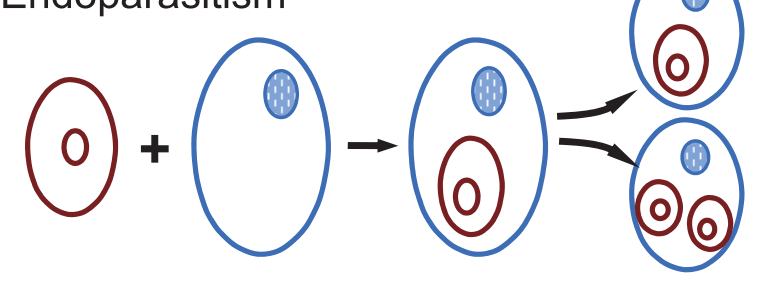

Fig. 1. Phagocytosis (engulfment and subsequent digestion of the red by the blue cell), parasitism (penetration of the blue by the red cell, propagation of the parasite on expenses of the host and eventual burst of the blue host cell), endosymbiosis (engulfment and gradual enslaving as an organelle of the red cell by the blue cell) and endoparasitism (penetration of the blue by the red cell and remaining/propagation of the endoparasite within the progeny of the host cell) as options for interaction between single cells. Rings inside endosymbiont or parasitic cells (red) represent either prokaryotic circular genomes or eukaryotic nuclei; blue circles in blue cells represent nuclei of eukaryotic hosts.
Sagan, 1986) or even nuclei. In these cases a (proto)eukaryotic cell engulfed a prokaryotic one and enslaved or domesticated it for tasks of the host cell. In this way, the engulfed cell is kept alive and propagated but has lost its physiological independence. Such unfriendly takeovers happened even among eukaryotic cells, as has been shown for some algae in the form of nucleomorphs, the degenerated remnants of the nuclei of endosymbiontic algal cells (for review see Archibald, 2007), located between the inner and outer membranes of a plastid. The gain of functionally specialized cellular organelles by endosymbiosis clearly contributes to the evolutionary success of eukaryotes. (Theoretically, a complementary event would be 'mild' endoparasitism, whereby a cell as a pathogen or parasite would penetrate another one, survive within the host cell without causing major damage, and become transferred to offspring cells during host cell division for a series of generations.)

Contrary to the before-mentioned cellular interactions, sexuality is an indirectly reversible and symmetric interaction, sustainable by reiteration, between equal-ranking (but not identical) cells (Fig. 2). Two cells (of opposite mating type) fuse and merge their nuclear genomes (immediately or after some mitotic cycles as dikaryons in some fungi). Later on, the two parental genomes, combined within one nucleus, undergo meiosis, i.e. pairing of homologous parental chromosomes followed by two cell divisions and resulting in four daughter cells, each containing a complete genome, usually stitched together from components of either of the parental genomes (Fig. 2). In principle, two of such daughter cells may fuse again, finishing a sexual life cycle and starting a new one. The sexual life cycle represents an alternation of nuclear

\section{Sexuality with similar-shaped isogametes}

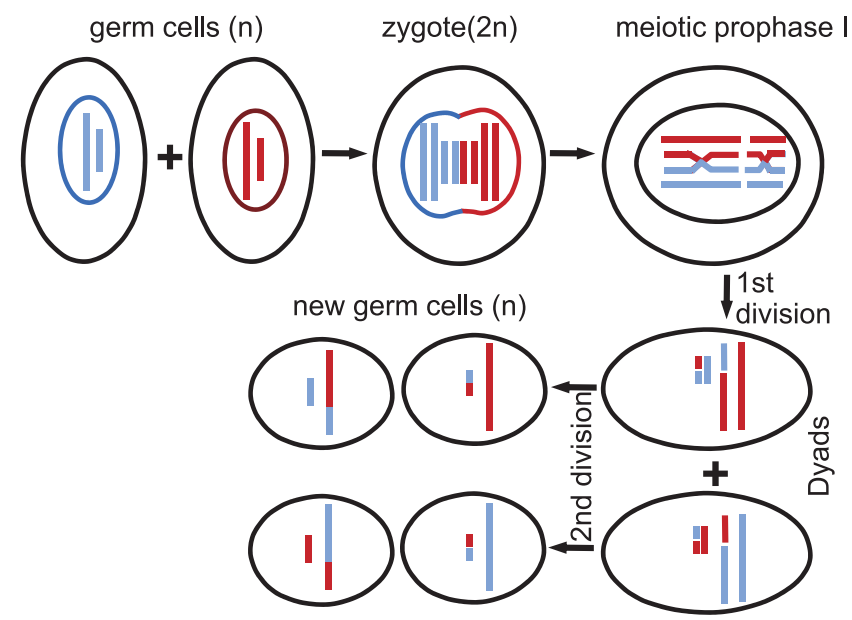

Fig. 2. Sexuality as option for interaction between single cells (for details see text). 
phases, i.e. between nuclei having one copy of the genome, as the haploid parental germ cell, and the diploid nuclei having the two parental genomes. During evolution, morphologically identical 'isogametes' evolved into differentiated male or female 'anisogametes'. Both the haploid and the diploid nuclear phases can be represented by multicellular bodies obtained by mitotic cell divisions. The time point at which parental germ cell nuclei fuse within the merged cell (or its descendants), and the relative as well as the absolute extension of the life span of the two generations with different nuclear phases (Fig. 3 and Greilhuber and Doležel, 2009) have changed in the course of evolution. The process of germ cell fusion is called fertilization (mixis) and the reductional re-sorting of parental nuclear genomes towards new germ cells is called meiosis.

Meiosis is apparently derived from mitotic nuclear division which occurs ubiquitously among eukaryotes (Wilkins and Holliday, 2009). Homologous proteins for all essential molecular components of mitosis already appear within prokaryotes (actins, tubulins and the SMC complex proteins for chromosome movement, cohesion and condensation, see Hirano, 2005; Erickson, 2007). Meiosis usually differs from mitosis in the following respects: i) Two successive nuclear divisions occur during meiosis. ii) One genome replication occurs before meiosis, creating a cell with four copies of the genome (two homologous chromosomes, each consisting of two identical sister chromatids), but no replication occurs between the two divisions. iii) During the first meiotic division, the two homologous chromosomes of each chromosome pair are separated into the daughter cells, reducing the number of
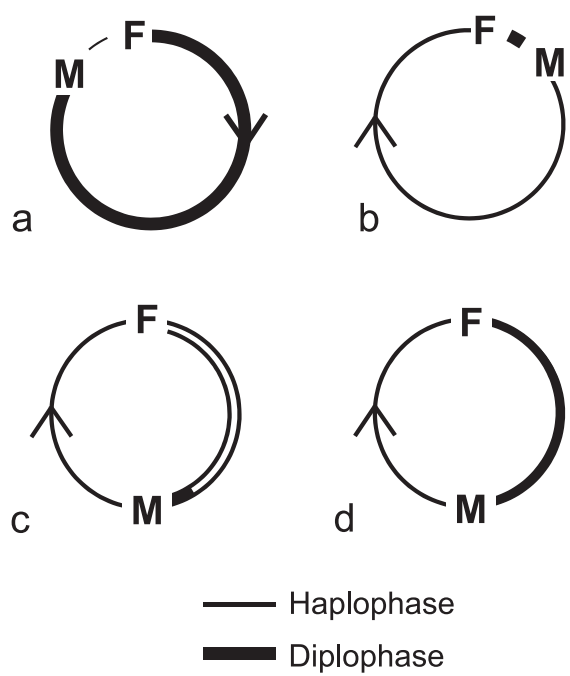

Fig. 3. The alternation between diploid and haploid nuclear phases in sexual eukaryotes. a diplonts (animals), b haplonts (many green algae), c dikaryohaplonts (fungi), d diplohaplonts (embryophyta), $\mathrm{F}$ fertilization, $\mathrm{M}$ meiosis (after Greilhuber and Doležel, 2009). chromosomes from diploid to haploid. iv) Sister chromatids are separated during the second division (except regions exchanged between homologous chromosomes by crossing over already during the first meiotic division). The essential new feature is pairing of homologues at the beginning of the first division and the 'monopolar-orientation' of both sister chromatids of each paired chromosome at metaphase I, allowing sister centromeres to be attached by spindle fibres from the same pole (Hauf and Watanabe, 2004). The synaptonemal complex aligns homologous chromosomes for recombination between nonsister chromatids, yielding cross overs which in turn seem to be responsible for tension at metaphase I, when spindle tubuli from opposite poles attach to homologous centromeres to resolve the check point control. Since genome doubling is possible without sexual fusion of cells, some kind of chromosome number reduction (Hurst and Nurse, 1991) might have evolved e.g. in endomitotic diploids before 'true' sex emerged. True meiosis has to adopt homologue pairing, which also occurs in somatic cells of some animals (e.g. Drosophila). Between both meiotic divisions 'replication licensing' (allowance for another round of replication after nuclear division) has to be suppressed, likely by prevention of sister centromere separation (Wilkins and Holliday, 2009). During chromosome pairing (synapsis) in prophase I, non-sister chromatids of homologues undergo symmetric reciprocal exchanges (crossing over). This exchange is based on a mechanism of homologous recombination repair that already occurs in prokaryotes at sites of double strand breaks (DSBs). The result is an exchange of paternal and maternal DNA between homologues, generating new combinations, in addition to the re-sorting of parental homologues during the reductional first meiotic division and to the new combination of alleles via fertilization. Because segregation of parental homologues in meiosis I is a stochastic process, the probability that germ cells contain the same chromosome sets as the germ cells of the previous generation decreases with increasing chromosome numbers. Even in case of only one chromosome pair, the occurrence of cross-over(s) would prevent germ cells genetically identical to the parental ones.

Ancestral, atypical or derived forms of sexuality Parasexuality, as non-reciprocal exchange of genetic material (plasmids) occurs between prokaryotes via a transient cell fusion (conjugation). Sex among some unicellular eukaryotes, for instance ciliates, consists of reciprocal exchange (and subsequent fusion) of haploid micronuclei during a transient cell conjugation (e.g. Jonsson et al., 2009 and Fig. 4). Some eukaryotes have lost or modified sexuality. Some animals generate progeny via parthenogenesis (unisexual development of female gametes without fertilization). In several higher plants various forms of asexual seed formation (apomixis) 


\section{Sex by conjugation and exchange of micronuclei (Ciliates)}

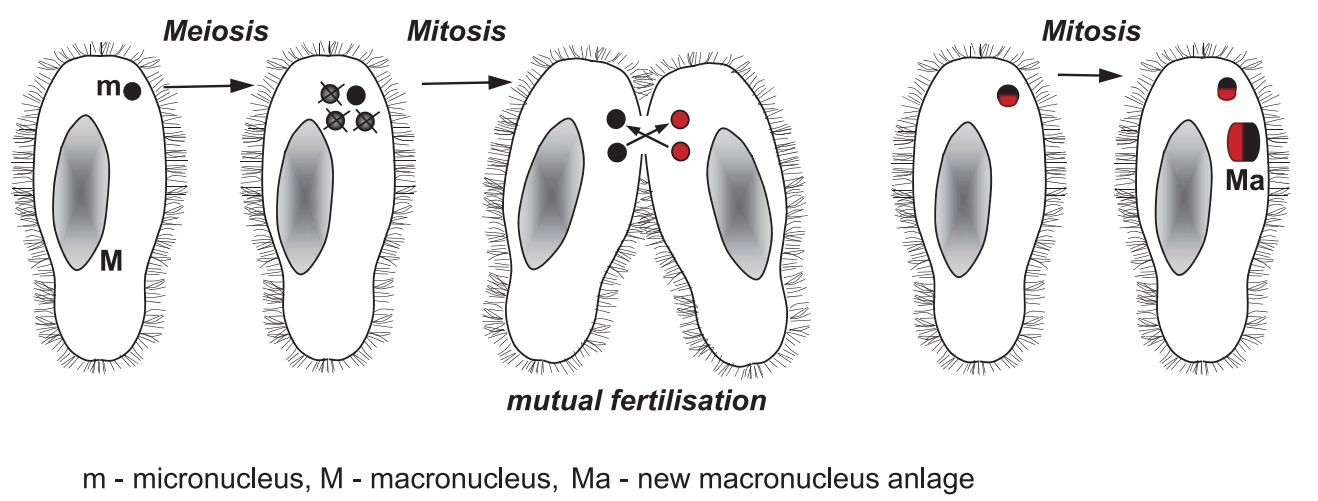

Fig. 4. Sex by conjugation and exchange of micronuclei (modified after Jonsson et al., 2009).

have evolved. Nevertheless, most apomictic plants are pseudogamous facultative apomicts, i.e., they produce male gametes that fertilize the nucleus of the female central cell (from which the nutritive endosperm develops) but not the egg cell nucleus. Thus, pseudogamous facultative apomicts have retained some potential for sexuality (for review see Koltunow and Grossniklaus, 2003). Unisexual propagation can be beneficial for fast colonisation of new environmental niches. Because unisexually propagating organisms abandon the variability they would gain from sexuality, they can survive with asexual life style as long as the inherent variability or the potential to generate new variability suffices to adapt to the actual changes of their environment.

What is the evolutionary advantage of sexuality? Sexuality is complex and costly, for instance through the need of two sexes instead of one for generating progeny, the need for sexual partners and gametes of opposite sex find each other, and, in case of anisogamy, in many cases, by the loss of three of the four products arising from female meiosis (Maynard Smith, 1978). This raises the question as to the benefit(s) of sexuality (for a recent overview see e.g. Hollricher, 2009). At the first glance one could think, repair of double strand breaks and the correction of either deletion or insertions in one chromosome of a homologous pair via recombination during meiosis I, which requires homologous template sequences, might be an advantage of sexuality. However, i) the direction of correction of heteroduplexes would be at random either toward the wild-type or the mutant sequence, and ii) one must consider that all known repair mechanisms already occur in prokaryotes and take place in eukaryotes independently of meiosis, at least in mitotically active cells. Wilkins and Holliday (2009) argue that meiotic homologue pairing decreases rather than increases recombination frequency because recombination is restricted by pairing to allelic sequences and excludes recombination between ectopic homologous sequences, thus avoiding genome destabilization. Genome destabilization is a frequent consequence of recombination repair involving paralogous sequences (i.e., homologous repeats in ectopic positions). Suppression of non-allelic exchange during meiosis is achieved by scheduled induction of DSBs via endonucleases, and recombination repair using only allelic sequences of paired homologous chromosomes as a template. Thus, the meiotic recombination between homologues leads to cross-overs, keeping homologues together to ensure correct segregation at anaphase I, and, in addition, seems to be devoted to the generation of new allele combination rather than to repair DNA damage. In somatic cells on the other hand, recombination repair is an error-prone late step in the hierarchy of repair tools, occurring when other mechanisms are exhausted or cannot cope with the damage. The main lesion eliciting recombination repair are DSBs that arise either directly or via interference of gaps, resulting from excision repair processes, with replication mediated strand discontinuities (Schubert et al., 2004). Unrepaired DSBs are lethal for dividing cells. DSB repair, except for non-homologous end joining, requires at least a limited extent of homologous sequence as a template. Without a strict alignment of homologous allelic sequences (of sister chromatids or of homologous chromosomes), in large genomes with a high degree of sequence redundancy, ectopic homologous regions may serve as template for homologous recombination repair and lead to aberrant chromosome rearrangements such as deletions, duplications or inversions within, and symmetric or asymmetric reciprocal translocations between individual chromosomes. Thus, in contrast to meiotic recombination, homologous recombination repair and non-homologous end-joining in somatic cells provide a chance for 'last minute' rescue from lethal DSBs at the risk of a high frequency of incor- 
rect repair. Repair that does not precisely restore the original situation causes variability, i.e. mutations that might be deleterious, neutral, or, in rare cases, advantageous.

Sexuality displays beneficial traits for the cells (organisms) involved:

1. The varying extension of the alternating ploidy phases in different branches of eukaryotes (Fig. 3) might be an adaptation to a different degree of environmental selection pressure: While the haploid generation undergoes strong selection against deleterious mutations and favours an immediate selection of beneficial mutations (speeding up the evolution in varying environments), the diploid generation assures against loss of essential gene functions (Otto and Gerstein, 2008).

2. Combination of similar but not identical genetic information generates increased adaptability by meiotic recombination and re-sorting between the parental genomes (Goddard et al., 2005). The high adaptability refers to the fast spread of positive variation, to the elimination of slightly harmful mutations and to a balanced evolutionary race in host-parasite relationship as well.

3. The high variability caused i) by meiotic resorting of the parental chromosome complements into the next germ cell generation, ii) by recombination between paired homologues, and iii) by new combination of alleles via fertilization, contrary to environmental mutagenesis, does seldom generate deleterious allele combinations. ('Combination breeding' also proved to be more successful than 'mutation breeding'.) Combination of deleterious alleles mostly leads to elimination of mutationally laden chromosomes from the genetic pool, while the complementary ('de novo') combination of advantageous alleles favours transmission of the carrier chromosome to the progeny (Kondrashov, 1988). Often, selectively neutral allele combinations are propagated by sexual organisms and can be used as 'raw material' for later adaptive evolution, or new combinations themselves are advantageous (e.g. Barton and Charlesworth, 1998).

4. The development of different complementary strategies of the sexual partners (few large, sessile and resource-rich female gametes on the one hand and many small, highly motile male gametes on the other) make sure optimal equipment (female) complemented by wide distribution for frequent fertilization (male) of gametes (Parker et al., 1972) and thus optimal propagation of the genes of both sexes.

5. Sexual dimorphism in multicellular organisms enables complementary task division and minimum competition of the different sexes for the same resources. Apparently, the benefits of germ cell and/ or organismic sexual dimorphism outweigh potential hazards that dimorphism may bear for male or female sexes in distinct cases. (Some female spiders eat their mates after copulation.)

6. Sexual attractiveness between germ cells at the chemical level and between multicellular organisms additionally at the neurophysiological level (by appetent behaviour for and innate responses to morphological triggers), enforce maintenance of sexuality.

Such benefits might have contributed to origin and/or maintenance of sexuality.

Sexual exchange requires a compatibility of the genetic systems of both partners. Factors that reduce this compatibility, for instance, polyploidization, deviation of diploid chromosome numbers or gain or loss of relevant alleles, may generate sexual barriers, driving former partners into sympatric speciation processes via inbreeding.

Thus, independent of the so-called 'battle' of sexes for expression (and eventual propagation) of paternal or maternal alleles in the offspring (Reik and Walter, 2001), sexuality is the only highly balanced, mutually beneficial, self-improving and cooperative interaction between equalranking cellular (and organismic) partners with a similar genetic equipment. Once established, it seems to fit best the joint interest of sexual partners to optimize propagation of their genes when varying environments require a high adaptability.

I thank Konrad Bachmann, Johann Greilhuber, Josef Loidl, Fritz Matzk, Gudrun Mönke, Elvira Hörandl and Tim Sharbel for stimulating discussions.

\section{REFERENCES}

Archibald, J. M. (2007) Nucleomorph genomes: structure, function, origin and evolution. BioEssays 29, 392-402.

Barton, N. H., and Charlesworth, B. (1998) Why sex and recombination? Science 281, 1986-1990.

Erickson, H. P. (2007) Evolution of the cytoskeleton. BioEssays 29, 668-677.

Goddard, M. R., Godfray, H. C., and Burt, A. (2005) Sex increases the efficacy of natural selection in experimental yeast populations. Nature 434, 636-640.

Greilhuber, J., and Doležel, J. (2009) 2C or not 2C: a closer look at cell nuclei and their DNA content. Chromosoma 118 , 391-400.

Hauf, S., and Watanabe, Y. (2004) Kinetochore orientation in mitosis and meiosis. Cell 119, 317-327.

Hirano, T. (2005) SMC proteins and chromosome mechanics: from bacteria to humans. Philos. Trans. R. Soc. Lond. B Biol. Sci. 360, 507-514.

Hollricher, K. (2009) Evolution of sexual reproduction. Lab Times 5-2009, 24-29.

Hurst, L. D., and Nurse, P. (1991) A note on the evolution of meiosis. J. Theor. Biol. 150, 561-563.

Jonsson, F., Postberg, J., and Lipps, H. J. (2009) The unusual way to make a genetically active nucleus. DNA Cell Biol. 28, 71-78.

Koltunow, A. M., and Grossniklaus, U. (2003) Apomixis: a devel- 
opmental perspective. Annu. Rev. Plant Biol. 54, 547-574.

Kondrashov, A. S. (1988) Deleterious mutations and the evolution of sexual reproduction. Nature 336, 435-440.

Margulis, L., and Sagan, D. (1986) Origins of Sex: Three Billion Years of Recombination. Yale Univ. Press, New Haven.

Maynard Smith, J. (1978) The Evolution of Sex. Cambridge Univ. Press, Cambridge.

Mereschkowsky, C. (1905) Über Natur und Ursprung der Chromatophoren im Pflanzenreiche. Biol. Zentbl. 25, 593-604.

Ohno, S. (1970) Evolution by Gene Duplication. Springer, BerlinHeidelberg-New York.

Otto, S. P., and Gerstein, A. C. (2008) The evolution of haploidy and diploidy. Curr. Biol. 18, R1121-1124.

Parker, G. A., Baker, R. R., and Smith, V. G. (1972) The origin and evolution of gamete dimorphism and the male-female phenomenon. J. Theor. Biol. 36, 529-553.

Reik, W., and Walter, J. (2001) Evolution of imprinting mechanisms: the battle of the sexes begins in the zygote. Nature Genet. 27, 255-256.

Schubert, I. (1988) Eukaryotic nuclei of endosymbiontic origin? Naturwissenschaften 75, 89-91.

Schubert, I., Pecinka, A., Meister, A., Schubert, V., Klatte, M., and Jovchev, G. (2004) DNA damage processing and aberration formation in plants. Cytogenet. Genome Res. 104, 104-108.

Wilkins, A. S., and Holliday, R. (2009) The evolution of meiosis from mitosis. Genetics 181, 3-12. 